\title{
Silencing Suppressor Protein VPg of a Potyvirus Interacts With the Plant Silencing-Related Protein SGS3
}

\author{
Minna-Liisa Rajamäki, Janne Streng, and Jari P. T. Valkonen \\ Department of Agricultural Sciences, P.O. Box 27, Fl-00014 University of Helsinki, Finland
}

Submitted 11 April 2014. Accepted 16 July 2014.

\begin{abstract}
Viral genome-linked protein (VPg) of potyviruses is involved in multiple steps of the potyvirus infection cycle, including viral multiplication and movement in plants. Recently, we showed that VPg of Potato virus A (PVA; genus Potyvirus) suppresses sense-mediated RNA silencing, which is linked to one or both nuclear or nucleolar localization. Here, we studied interactions between VPg and components of the plant RNA silencing pathway. Results showed that VPg interacts with the SGS3 protein of Solanum tuberosum and Arabidopsis thaliana, as shown by yeast two-hybrid analysis and bimolecular fluorescence complementation assays. VPg-SGS3 interactions co-localized with small cytoplasmic bodies that contained plant RNA-dependent RNA polymerase 6 (RDR6) (likely SGS3/RDR6 bodies). The N-terminal zinc finger (ZF) domain of SGS3 was the main determinant of the VPg interaction. Our data also suggest that the ZF domain controls SGS3 localization. SGS3 homodimerization was controlled by multiple protein regions. The VPg-SGS3 interaction appeared beneficial for PVA, as viral RNA levels correlated positively with sgs3 mRNA levels in the SGS3-silenced and SGS3-overexpressing leaves of Nicotiana benthamiana. The data support the idea that VPg acts as a suppressor of RNA silencing and suggest that an interaction with SGS3 may be important, especially in suppression of sense-mediated RNA silencing.
\end{abstract}

RNA silencing is an important mechanism for regulating gene expression in eukaryotes and also functions in antiviral defense (Pumplin and Voinnet 2013). RNA silencing is triggered by double-stranded RNA (dsRNA), which is cleaved into small interfering RNAs (siRNAs) 21 to 24 nucleotides (nt) in length by dicer-like endoribonucleases in plants. The dssiRNA is loaded into an RNA-induced silencing complex containing an RNaseH-like argonaute (AGO) enzyme and one of the siRNA strands is used to guide AGO to cleave a homologous RNA molecule (Pumplin and Voinnet 2013).

RNA silencing induced by the overexpression of gene transcripts (sense-mediated silencing) requires the activity of RNA-dependent RNA polymerases (e.g., RDR6) for conversion of single-stranded RNA (ssRNA) to dsRNA (Béclin et al.

Sequence data is available in the GenBank and EMBL databases under accession numbers HG970650 (StSGS3a) and HG970651 (StSGS3b).

Corresponding author: M.-L. Rajamäki; E-mail: minna.rajamaki@helsinki.fi

* The $e$-Xtra logo stands for "electronic extra" and indicates that seven supplementary figures and one supplementary table are published online and that Figure 2 appears in color online.

(C) 2014 The American Phytopathological Society
2002; Mourrain et al. 2000). In addition, a plant-specific RNAbinding protein, suppressor of gene silencing 3 (SGS3), is required for this process (Béclin et al. 2002; Mourrain et al. 2000). RDR6 and SGS3 are also needed for the amplification of RNA silencing via the production of secondary siRNAs and for the production of endogenous trans-acting small interfering RNAs (ta-siRNAs), and natural antisense transcriptderived siRNAs (Peragine et al. 2004; Vaucheret, 2006; Yoshikawa et al. 2005, 2013).

The function of SGS3 is interlaced with that of RDR6 but is poorly understood (Mourrain et al. 2000; Yoshikawa et al. 2005). SGS3 binds dsRNA containing $5^{\prime}$ overhangs with high affinity but binds poorly to dsRNA with blunt-ends or $3^{\prime}$ overhangs (Fukunaga and Doudna 2009). SGS3 contains a zinc finger (ZF) domain, an XS domain (XS) similar to the rice gene $\mathrm{X}$ product, and a coiled-coil (CC) domain. XS is needed for RNA binding (Fukunaga and Doudna 2009; Zhang and Trudeau 2008), whereas the CC domain is involved in the homodimerization of SGS3 (Elmayan et al. 2009). The function of the ZF domain is not known.

SGS3 localizes to small cytoplasmic granules, and the XS and $\mathrm{CC}$ domains are important for this localization pattern (Kumakura et al. 2009). In addition, SGS3 can be found in the nucleoplasm, in which it is enriched within a round perinucleolar structure (Pontes et al. 2013). SGS3 co-localizes and interacts with RDR6, which is observed in small cytoplasmic bodies, designated as SGS3/RDR6 bodies or siRNA bodies (Kumakura et al. 2009). SGS3/RDR6 bodies are different from the processing bodies ( $\mathrm{P}$ bodies) that contain components of mRNA turnover (Kumakura et al. 2009). The RNase H-like endonuclease AGO7 is involved in ta-siRNA biogenesis and co-localizes with SGS3/RDR6 bodies (Jouannet et al. 2012).

Viruses have evolved to counteract RNA silencing by encoding proteins that interfere with various steps of the RNA silencing pathway. Some viral proteins target host silencing pathway proteins. Many viral silencing suppressor proteins target AGO to inhibit its slicing activity or to induce its degradation (Azevedo et al. 2010; Bortolamiol et al. 2007; Chiu et al. 2010; Giner et al. 2010; Gonzalez et al. 2010; Zhang et al. 2006). However, the V2 protein of Tomato yellow leaf curl virus (TYLCV; ssDNA genome, genus Begomovirus) and the p2 protein of Rice stripe virus (RSV; negative-sense ssRNA genome, genus Tenuivirus) interact with SGS3 and interfere with sense RNA silencing (Du et al. 2011; Glick et al. 2008). The P6 protein of Rice yellow stunt virus (negative-sense ssRNA genome, genus Rhabdovirus) binds RDR6 to interfere with the production of secondary siRNAs and systemic RNA silencing (Guo et al. 2013).

Potyviruses (genus Potyvirus, family Potyviridae) constitute the largest group of plant-infecting RNA viruses (Adams et al. 2012). Recently, we found that the viral genome-linked protein 
(VPg) of Potato virus A (PVA) interferes with RNA silencing in host plants (Rajamäki and Valkonen 2009) in addition to HCpro, which is a well-known potyvirus suppressor of RNA silencing (Anandalakshmi et al. 1998; Brigneti et al. 1998; Jamous et al. 2011; Kasschau and Carrington 1998; Szittya and
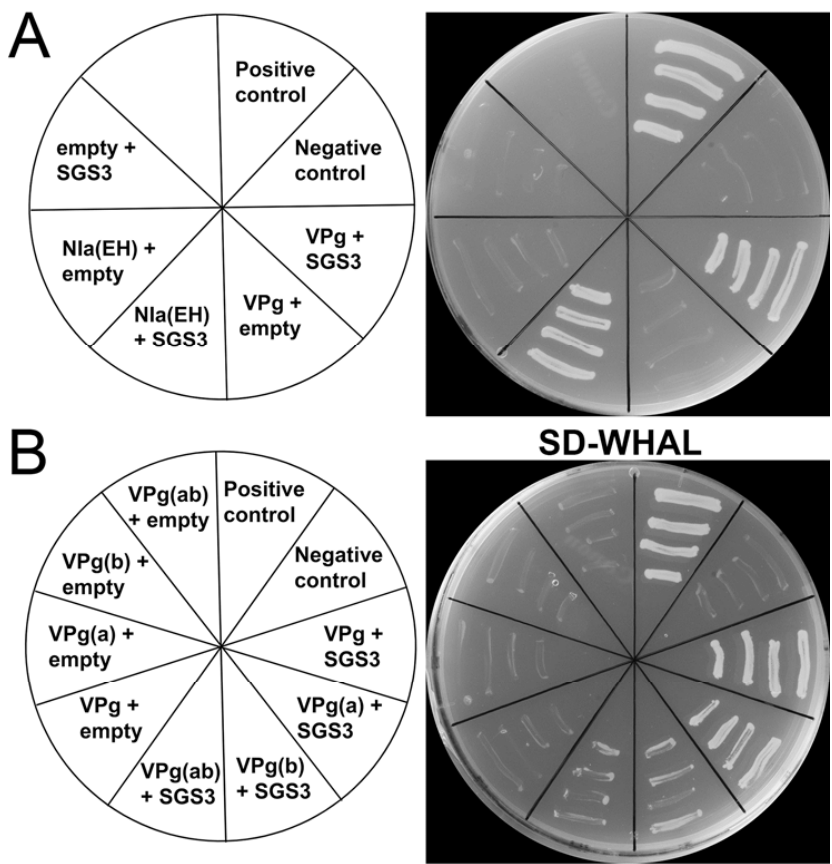

$B^{\prime}$
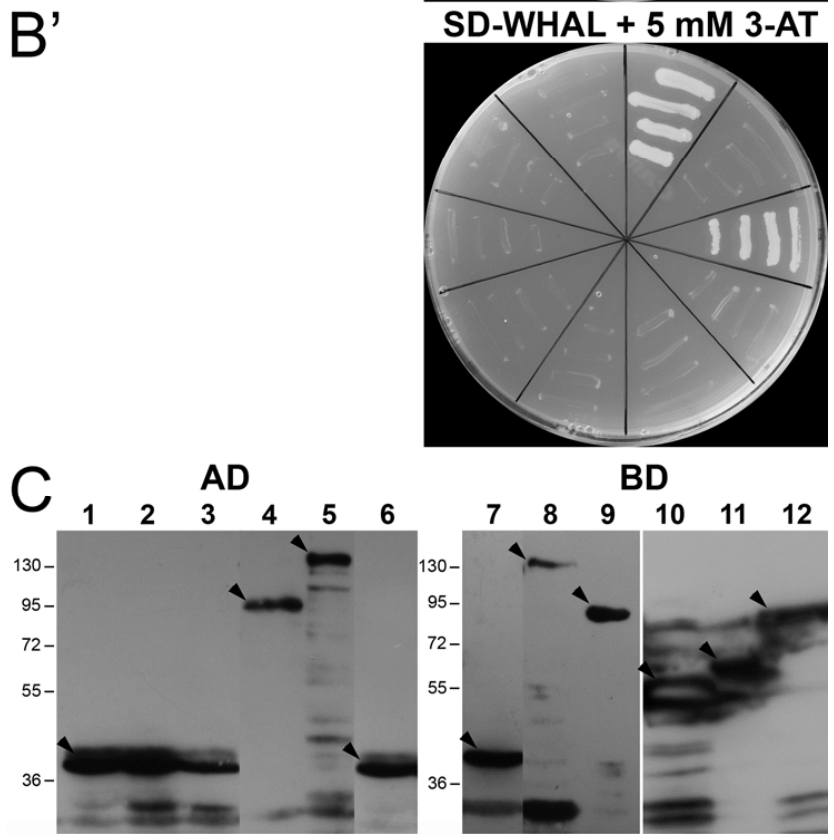

BD

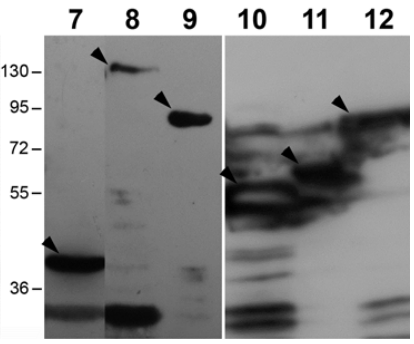

Fig. 1. Interactions of Potato virus A (PVA) viral genome-linked protein (VPg) and mutants of VPg with AtSGS3 in our yeast two-hybrid system. A, Interactions of $\mathrm{VPg}$ and $\mathrm{NIa}(\mathrm{EH})$ with AtSGS3 as detected based on yeast growth on stringent selection media (SD-WHAL) 2 days after plating. $\mathrm{NIa}(\mathrm{EH})$ contains the mutation $(\mathrm{E} 189 \mathrm{H})$ that prevents internal proteolytic processing between VPg and NIa-Pro (Rajamäki and Valkonen 2009). B, Mutations that compromise the nuclear and nucleolar localization of $\mathrm{VPg}$ reduce interaction of $\mathrm{VPg}$ with AtSGS3, as determined by slower growth of yeast on SD-WHAL media 2 days after plating and B', a lack of yeast growth on the media containing $5 \mathrm{mM} 3$-amino-1,2,4-triazole (3-AT) 5 days after plating. $\mathbf{C}$, Immunodetection of the expressed proteins in yeast using monoclonal antibodies specific to the activation domain (AD) or the binding domain (BD). Lane 1, VPg; 2, VPg(a); 3, VPg(b); 4, AtSGS3; 5, AtRDR6; 6, VPg(ab); 7, VPg; 8, AtRDR6; 9, AtSGS3; 10, ZF-AtSGS3; 11, XSCC-AtSGS3; and 12, AtSGS3. The sizes of molecular mass markers (kDa) are shown to the left.
Burgyan 2013). VPg is a multifunctional protein involved in viral replication and movement (Rajamäki et al. 2004). In virusinfected cells, VPg and its precursor protein, the nuclear inclusion protein a (NIa), are typically localized to the nucleus with the aid of a strong bipartite nuclear localization signal (NLS) at the N-proximal region of $\mathrm{VPg}$ (Rajamäki and Valkonen 2009). Nuclear or nucleolar localization of VPg was found to correlate with its function as a silencing suppressor (Rajamäki and Valkonen 2009). VPg suppresses sense-mediated gene silencing but is unable to suppress RNA silencing induced by dsRNA (e.g., hairpin constructs) (Germundsson et al. 2007; Rajamäki and Valkonen 2009), which suggests that VPg targets a step of RNA silencing required for the generation of dsRNA by plant RDR6. However, the mechanism by which VPg suppresses RNA silencing is not known. Therefore, we investigated VPg interactions with SGS3 and RDR6 to aid in the understanding of the mechanism by which VPg interferes with RNA silencing.

\section{RESULTS}

PVA VPg interacts with Arabidopsis SGS3 but not RDR6.

PVA VPg suppresses only sense-mediated RNA silencing. Therefore, the interactions of VPg with RDR6 and SGS3 required for the generation of dsRNA were tested, using the yeast two-hybrid system (YTHS). No interaction was detected between VPg and the RDR6 of Arabidopsis thaliana (AtRDR6) (Supplementary Fig. 1A), whereas an interaction between VPg and SGS3 of A. thaliana (AtSGS3) was readily detected, indicated by robust growth on a stringent selection medium (Fig. 1A). An interaction between VPg and AtSGS3 was detected when VPg was expressed fused to the activation domain (AD) and AtSGS3 fused to the DNA binding domain (BD) in YTHS. Furthermore, when NIa (the precursor of VPg) was fused to the AD, it also interacted with AtSGS3 (Fig. 1A). Negative controls (VPg, NIa, and AtSGS3 tested against insertless, "empty" yeast plasmids) showed no growth (Fig. 1). The expression of each fusion protein was readily detected in yeast cells by Western blot analysis (Fig. 1C).

The $\operatorname{VPg}$ mutants $[\operatorname{VPg}(a), \operatorname{VPg}(b)$, and $\operatorname{VPg}(a b)]$, in which the nuclear and nucleolar localization and the ability to suppress RNA silencing are compromised (Rajamäki and Valkonen 2009), were subsequently tested for interactions with AtSGS3 in YTHS. While the mutants were able to interact with AtSGS3 to some extent, slightly slower yeast growth indicated that their interactions with AtSGS3 were weaker than those observed with wild-type VPg (Fig. 1B). Application of $5 \mathrm{mM} 3$-amino1,2,4-triazole (3-AT) to the selection media totally abolished interactions of $\operatorname{VPg}(\mathrm{a}), \operatorname{VPg}(\mathrm{b})$, and $\operatorname{VPg}(a b)$ with $\operatorname{AtSGS3}$, whereas interaction of wild-type VPg with AtSGS3 was unaffected (Fig. 1B'). 3-AT is a competitive inhibitor of the HIS3 selection marker, and thus, higher affinity binding between two proteins is required for yeast growth on a selective media containing 3-AT (Joung et al. 2000). The results suggested that the mutations that compromise RNA silencing suppression and $\mathrm{VPg}$ nuclear and nucleolar localization also affect the VPgSGS3 interaction.

\section{Interactions of PVA VPg with SGS3 localizes to small cytoplasmic bodies in planta.}

Potato (Solanum tuberosum L., family Solanaceae) is a natural host of PVA. Therefore, we polymerase chain reaction (PCR)-amplified potato cDNA sequences encoding SGS3. Two variants, designated StSGS3a and StSGS3b, were cloned and used in further experiments. The deduced amino acid (aa) sequences of StSGS3a and StSGS3b were 631 residues long and differed from each other at three positions (K62R, R118S, 
and P205L) (Supplementary Fig. 2). The proteins were 48.8 and $48.9 \%$ identical, respectively, to AtSGS3 and were 6 aa longer than AtSGS3. StSGS3a and StSGS3b were nearly identical (between 97.6 and 98.1\%) to SGS3 of tomato (S. lycopersicum L., family Solanaceae), a functional homolog of AtSGS3 (Glick et al. 2008).

To test the interaction of PVA VPg with SGS3 in plant cells, bimolecular fluorescence complementation assay (BiFC) was used, as described by Rajamäki and Valkonen (2009). StSGS3a and StSGS3b were translationally fused at their C-termini with either the $\mathrm{N}$-terminal $\left(\mathrm{YN}_{1-154}\right)$ or the $\mathrm{C}$-terminal half $\left(\mathrm{YC}_{155-239}\right)$ of yellow fluorescent protein (YFP). Furthermore, YN or YC was fused to the $\mathrm{N}$ - or C-terminus of VPg and AtSGS3. The $\mathrm{YN}$ - and YC-tagged fusion proteins were coexpressed in the leaf epidermal cells of Nicotiana benthamiana by agroinfiltration, and signals of interaction were detected using epifluorescence and confocal microscopy 2 and 3 days postinfiltration (dpi). Interactions between VPg (YC-VPg or VPg-YC) and StSGS3aYN, StSGS3b-YN, and AtSGS3-YN were evident (Fig. 2; Supplementary Fig. 3A). The fluorescent signals were concentrated in small motile bodies distributed throughout the cytoplasm (Fig. 3A). Co-infiltration of VPg (YN-VPg or VPg-YN) and StSGS3a-YC, StSGS3b-YC, or AtSGS3-YC gave similar results (Fig. 2), whereas no interaction between VPg and YNAtSGS3 or YC-AtSGS3 was detected. Interactions between StSGS3 and VPg and between AtSGS3 and VPg were deemed to be specific, because fluorescent signals were not observed when StSGS3-YC, StSGS3-YN, AtSGS3-YC, or AtSGS3-YN were tested for interactions with potato eIF4E (Fig. 2G and H). In subsequent experiments, C-terminally tagged StSGS3 (StSGS3a-YN or StSGS3a-YC) and AtSGS3 (AtSGS3-YN and AtSGS3-YC) were used.

\section{VPg-SGS3 interactions in plant cells co-localize with cytoplasmic bodies containing RDR6.}

The fluorescent signals from the VPg-SGS3 interaction were concentrated in bodies that resemble the previously reported SGS3/RDR6 bodies (Kumakura et al. 2009). Therefore, interactions of AtRDR6 with SGS3 and VPg were studied. Interactions between StSGS3-YC and AtRDR6-YN and between AtSGS3-YC and AtRDR6-YN were observed in small fluorescent bodies in the cytoplasm (Fig. 3B) as has been reported previously with AtSGS3 and AtRDR6 (Kumakura et al. 2009), but no interaction was observed between VPg and AtRDR6. Co-localization of the VPg-SGS3 interaction with AtRDR6 was tested further by coexpressing VPg-YC, AtSGS3-YN, and AtRDR6, which was tagged with monomeric red fluorescence protein (AtRDR6-mRFP). The fluorescent bodies resulting from an interaction between VPg and SGS3 co-localized with fluorescence from AtRDR6-mRFP (Fig. 4A), suggesting that the VPg-SGS3 interaction occurs within the SGS3/RDR6 bodies (Kumakura et al. 2009).

An infectious PVA clone was engineered to express VPg$\mathrm{YC}$ from the NIb and coat protein (CP) junction of the viral polyprotein (PVA-VPgyc; Supplementary Fig. 4). In PVAVPgyc, VPg-YC is released from the viral polyprotein by cleavage of the flanking proteolytic cleavage sites of the viral NIa proteinase. Plants of $N$. benthamiana were inoculated with PVA-VPgyc by agroinfiltration and were tested with PVAspecific monoclonal antibodies by double-antibody sandwich enzyme-linked immunosorbent assay (DAS-ELISA) at $14 \mathrm{dpi}$. The inoculated plants were infected systemically, and the leaves contained high virus titers $(15.0 \pm 5.8 \mu \mathrm{g}$ of PVA CP per gram of fresh weight of leaf tissue). Infected plants displayed severe symptoms of malformation and chlorosis in systemically infected leaves, as observed with wild-type PVA. Subsequently, PVA-VPgyc and StSGS3-YN (or AtSGS3-YN) were coexpressed in the leaves of $N$. benthamiana by agroinfiltration. Interaction between $\mathrm{VPg}-\mathrm{YC}$ produced by multiplying PVA-VPgyc and the transiently expressed StSGS3-YN (and AtSGS3-YN) was observed in small fluorescent cytoplasmic bodies at 2 to $3 \mathrm{dpi}$, which was similar to the VPg-SGS3 interaction observed previously (Figs. 2 and 3A). These data showed that VPg and SGS3 interact in cytoplasmic bodies in PVAinfected cells.

\section{VPg-SGS3 interaction bodies are different from the replication vesicles of PVA.}

An engineered clone of PVA (PVA-6krfp), which expresses the 6K2-mRFP protein from the NIb and $\mathrm{CP}$ junction, was used to localize the viral replication vesicles induced by $6 \mathrm{~K} 2$ (Ala-Poikela et al. 2011). PVA-6krfp infected N. benthamiana plants systemically, as tested by DAS-ELISA at 14 dpi (1.6 \pm $0.4 \mu \mathrm{g}$ of PVA CP per gram of fresh weight of leaf tissue). PVA-6krfp was introduced jointly with VPg-YC and StSGS3YN (or AtSGS3-YN) into the leaf tissue of $N$. benthamiana by agroinfiltration, and signals indicating an interaction between VPg-YC and StSGS3-YN (and AtSGS3-YN) were detected in small cytoplasmic bodies, which co-localized with mRFP signals from 6K2-mRFP only occasionally (Fig. 4B). The mRFP signals, which indicated viral replication vesicles, were mainly associated with chloroplasts (Fig. 4B). The data suggested that VPg-SGS3 interactions do not occur in viral replication vesicles.

\section{ZF domain of SGS3 is the main determinant of VPg interaction.}

Constructs used for the expression of the truncated forms of SGS3 were prepared based on its predicted domain structure. ZF-SGS3 (aa 1 to 289) contains the N-terminal portion of SGS3, including a putative ZF domain. XSCC-SGS3 (aa 290 to 625) contains the XS and CC domains of SGS3, which are important for dsRNA binding and granule-like localization of SGS3 (Fukunaga and Doudna 2009; Kumakura et al. 2009) (Fig. 5A). A strong VPg-ZF-AtSGS3 interaction, similar to the
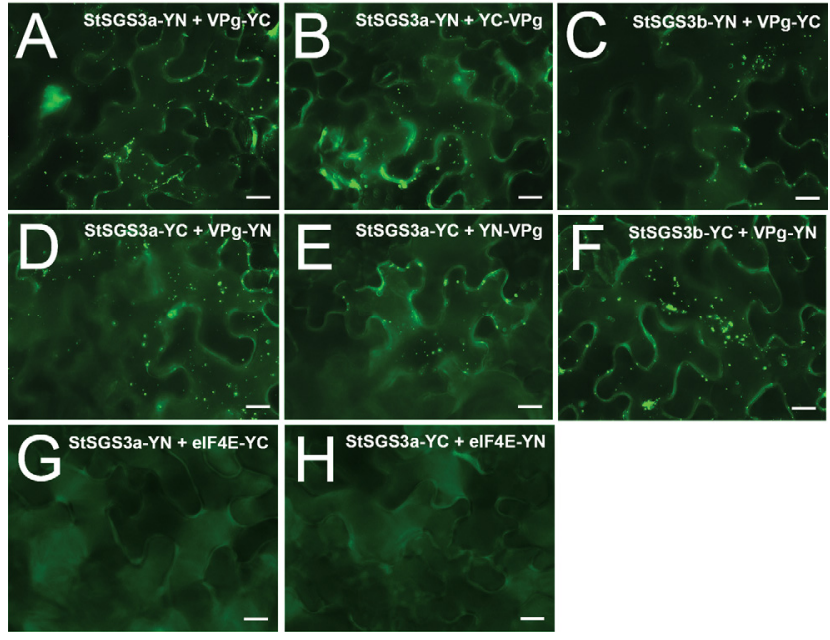

Fig. 2. Detection of interactions between Potato virus $A$ (PVA) viral genome-linked protein (VPg) and two variants of potato SGS3 (StSGS3a and StSGS3b) in punctate bodies in epidermal cells of leaves of Nicotiana benthamiana using bimolecular fluorescence complementation. A to $\mathbf{F}$, VPg and StSGS3 fused to YC or YN (C-proximal or N-proximal halves of the yellow fluorescent protein [YFP] gene) interact in punctate bodies. G and $\mathbf{H}$, Coexpression of StSGS3 and eIF4E (negative control). Leaves were infiltrated with pairs of Agrobacterium strains expressing tester proteins tagged with the different halves of YFP. Images were taken using an epifluorescence microscope 3 days postinfiltration using the same exposure parameters. Scale bar $=20 \mu \mathrm{m}$. 
interaction of VPg with full-length AtSGS3, was detected using YTHS, whereas the other half of SGS3 showed no interaction with VPg (Fig. 5B).

$\mathrm{VPg}-\mathrm{YC}$ was found to interact with ZF-StSGS3-YN when tested by BiFC. The signals resulting from interaction of $\mathrm{ZF}$ StSGS3 with VPg were distributed throughout the cytoplasm, showing a lack of specific localization (Fig. 5C), which is in agreement with earlier findings indicating that the XS and CC domains of SGS3 are needed for the granule-like localization of SGS3 (Kumakura et al. 2009). XSCC-StSGS3 also interacted with VPg. However, the localization was different. The interaction of VPg with full-length SGS3 was exclusively detected in the small cytoplasmic bodies (in $100 \%$ of the 300 fluorescent cells analyzed) (Figs. 2 and 5F). However, analysis of 235 fluorescent cells showed that the interaction between
VPg and XSCC-StSGS3 was usually found in the nucleus and subnuclear bodies (in $44 \%$ of the cells in the nucleus only; in $53 \%$ of the cells in the nucleus and cytoplasm), whereas in $3 \%$ of the cells analyzed, XSCC-StSGS3-VPg interaction was detected only in cytoplasm (Fig. 5D and E). Similar results were obtained by co-infiltration of VPg-YN and ZF-StSGS3-YC or XSCC-StSGS3-YC. These data suggest that the ZF of SGS3 is the main determinant that retains the VPg and SGS3 interaction in the cytoplasm.

The putative ZF motif ( $\mathrm{X}_{2}$-Cys- $\mathrm{X}_{2,4}-$ Cys- $\mathrm{X}_{12}$-His- $\mathrm{X}_{3,4,5}-\mathrm{His}$, aa 226 to 249) of SGS3 was mutated in AtSGS3 by introducing two amino acid substitutions (C228A and C231A) (mutant AtSGS3-ZFmut). Signals resulting from the interaction between AtSGS3-ZFmut and VPg appeared consistently weaker in BiFC than those resulting from the interaction between AtSGS3 and
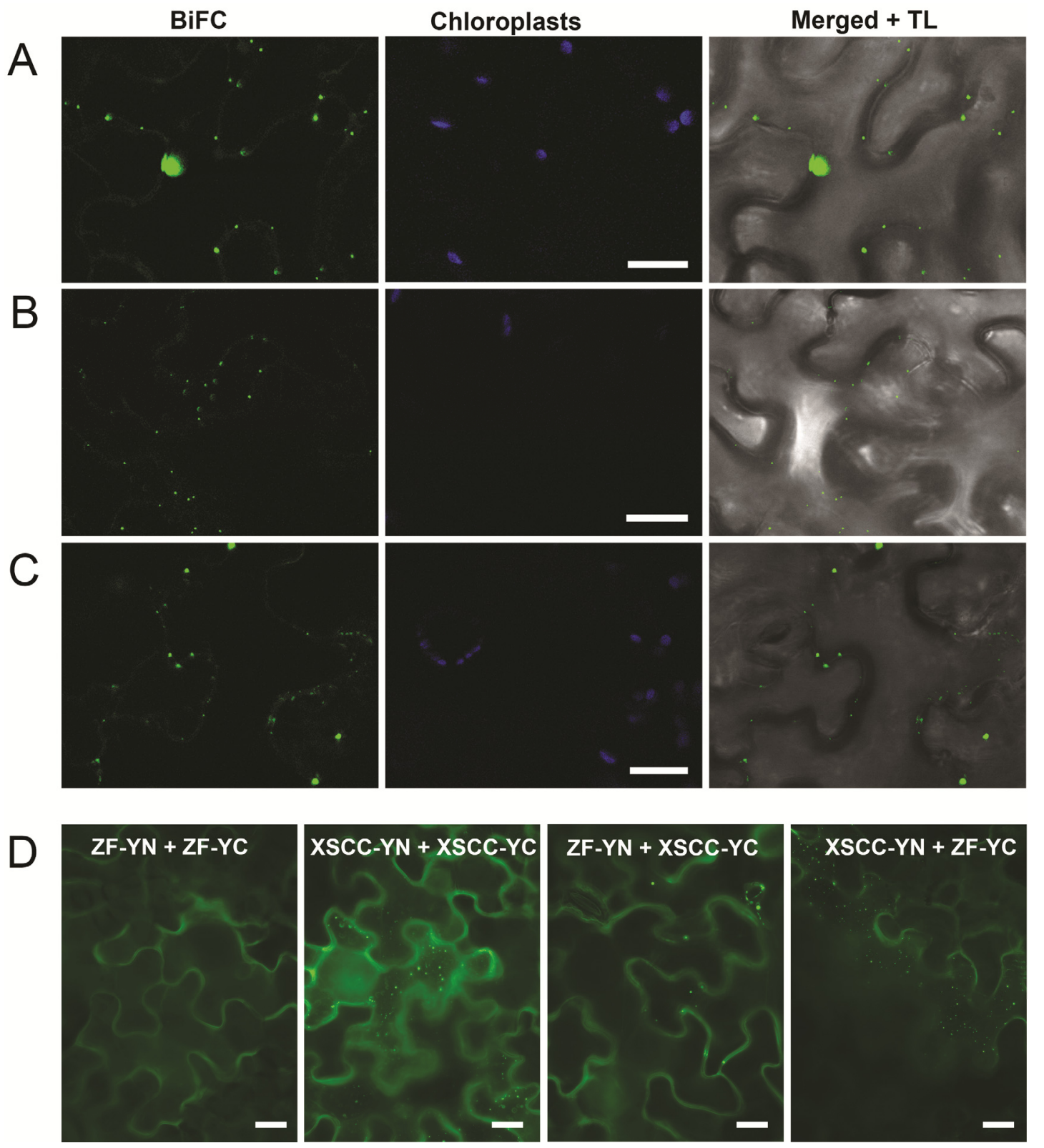

Fig. 3. Detection of protein-protein interactions in epidermal cells of leaves of Nicotiana benthamiana, using bimolecular fluorescence complementation (BiFC). Green fluorescence (BiFC) indicates A, interaction between viral genome-linked protein (VPg)-YC (C-proximal half of the yellow fluorescent protein [YFP]) and AtSGS3-YN (N-proximal half of YFP) (sizes of fluorescent bodies circa 0.5 to $6 \mu \mathrm{m}$ ), B, AtRDR6-YN and AtSGS3-YC (0.4 to $1.5 \mu \mathrm{m}$ ), and $\mathbf{C}$, AtSGS3-YN and AtSGS3-YC (0.5 to $2 \mu \mathrm{m})$. Chloroplasts are shown in blue. Images merged with transmitted light (far right panels) indicate the overall cell structures. The images in A to $\mathrm{C}$ are individual optical sections captured by confocal microscope 3 days postinfiltration. D, Interactions between truncated forms of StSGS3 as tested by BiFC with imaging performed under epifluorescence microscope 2 days postinfiltration. $\mathrm{ZF}=\mathrm{a}$ putative zinc finger domain; XSCC $=$ XS and coiled coil domains. Scale bars $=20 \mu \mathrm{m}$. 
VPg (Supplementary Fig. 5). However, while this finding further supports the importance of ZF in the interaction, Western blot analysis indicated that AtSGS3-ZFmut was expressed in lower amounts than AtSGS3, which may also explain the result (Supplementary Fig. 6). When AtRDR6 was coexpressed with ZF-SGS3 or XSCC-SGS3, no BiFC signals were detected, which suggests that both domains of SGS3 are important for interacting with AtRDR6.

\section{SGS3 self-interaction.}

SGS3 is thought to function as a homodimer and the CC domain of SGS3 is sufficient for homodimerization (Elmayan et al. 2009). We also detected the homodimerization of SGS3 in small cytoplasmic bodies by BiFC (Fig. 3C). The XSCCdomain construct of SGS3 self-interacted and, as with the fulllength SGS3, the resultant signals from the interaction were observed in small cytoplasmic bodies (Fig. 3D). An interaction between ZF-SGS3 and XSCC-SGS3 was detected in small cytoplasmic bodies, whereas the signals of ZF-SGS3 self-interaction were distributed throughout the cytoplasm and were not concentrated in granule-like bodies (Fig. 3D). Taken together, the results suggest that SGS3 has multiple sites that control SGS3 homodimerization. In addition, the results support the previous observation that the XSCC domain of SGS3 is required for the granule-like localization of SGS3 (Kumakura et al. 2009).

\section{PVA infection upregulates RDR6 expression}

but has no effect on expression of ta-siRNA target genes.

Besides antiviral defense, SGS3 and RDR6 are needed for the biogenesis of ta-siRNAs (Mourrain et al. 2000; Peragine et al. 2004; Yoshikawa et al. 2005). The possible influence of PVA infection on the expression of SGS3, RDR6, and the conserved TAS3 ta-siRNA target mRNAs (ARF [auxin-responsive factor] transcription factors) (Axtell et al. 2006) was examined. PVA was sap-inoculated to the two lowest leaves of five small tobacco plants in three independent experiments (Haikonen et al. 2013). Control plants were mock-inoculated with water. Carborundum was used as an abrasive. The first systemically infected leaves and the leaves at the same position in control plants were sampled at 12 days postinoculation. PVA infection in the samples was verified by immunocapture reverse tran-
scription-PCR (IC-RT-PCR). The results of quantitative realtime RT-PCR using gene-specific primers indicated that expression of RDR6 mRNA was induced in PVA-infected leaves, as compared with control plants (Fig. 6), whereas the expression of SGS3 and ARF mRNAs was not altered significantly compared with controls. Similar results were obtained by analysis of the leaf transcriptome with the Agilent tobacco gene expression $4 \times 44 \mathrm{k}$ microarray (Haikonen et al. 2013). RDR6 gene expression was slightly upregulated $(P$ value $<0.05)$ in leaves infected with PVA compared with the healthy control plants, whereas no significant differences could be detected with the ARF3 gene expression levels. These results suggested that PVA infection does not significantly affect TAS3 ta-siRNA biogenesis.

\section{Suppression of RNA silencing is not affected by overexpression of SGS3.}

VPg and green fluorescent protein (GFP) were coexpressed with StSGS3 or the truncated ZF-StSGS3 in the leaves of GFP-transgenic plants of $N$. benthamiana (line 16c) by agroinfiltration to test whether or not SGS3 interferes with or enhances the suppression of sense-mediated RNA silencing carried out by VPg. Coexpression of VPg and GFP enhanced GFP fluorescence in the infiltrated leaf areas, in contrast to the expression of GFP and the $\mathrm{VPg}(\mathrm{a})$ mutant (Supplementary Fig. 7), as shown previously (Rajamäki and Valkonen 2009). Coexpression of StSGS3 or ZF-StSGS3 with VPg and GFP had no impact on the brightness of GFP fluorescence. A red fluorescent zone appeared around all infiltrated regions at 8 to $9 \mathrm{dpi}$, indicating that local signaling of RNA silencing was not affected (Himber et al. 2003). These data indicated that overexpression of SGS3 does not interfere with or enhance silencing suppression by VPg.

\section{SGS3 silencing does not affect viral protein accumulation} but reduces the amount of viral RNA.

SGS3 was silenced in $N$. benthamiana using a Tobacco rattle virus (TRV)-based gene silencing vector carrying a fragment of the NbSGS3 gene (vector TRV-sgs3). As a control, plants were inoculated with a TRV vector carrying a fragment of the phytoene desaturase gene (TRV-PDS), which causes loss of chlorophyll and induces photobleaching as a visual sign
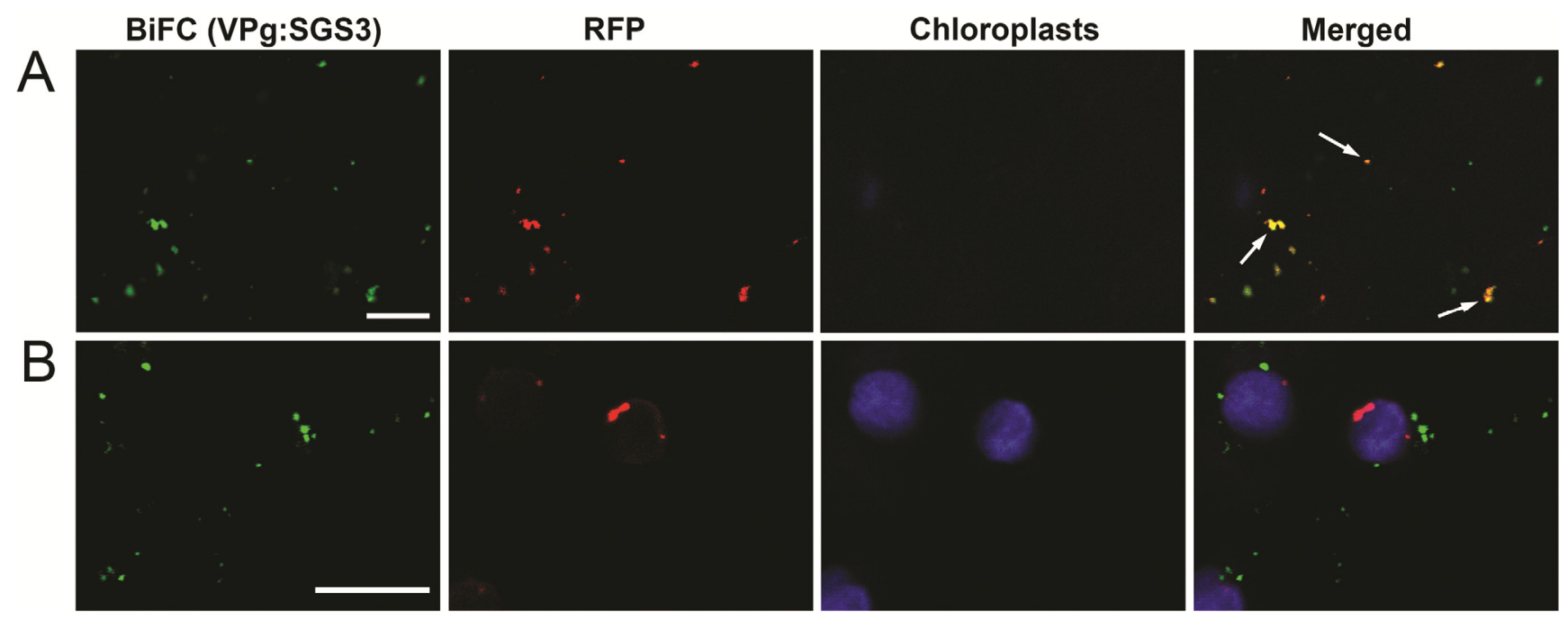

Fig. 4. Localization of the viral genome-linked protein (VPg)-AtSGS3 interaction in epidermal cells of leaves of Nicotiana benthamiana. A, VPg-AtSGS3 interactions as detected using bimolecular fluorescence complementation (BiFC) co-localized with AtRDR6-mRFP (monomeric red fluorescence protein). Arrows indicate position of some of the co-localized vesicles. B, 6K2-mRFP was expressed from the genome of Potato virus A (PVA) and red fluorescence was used to indicate the position of viral replication vesicles. The VPg-SGS3 interaction does not co-localize with 6K2-mRFP-derived viral replication vesicles in PVA-infected cells. The pictures are single optical sections captured by a confocal microscope 2 to 3 days postinfiltration. Scale bars $=10 \mu \mathrm{m}$. 


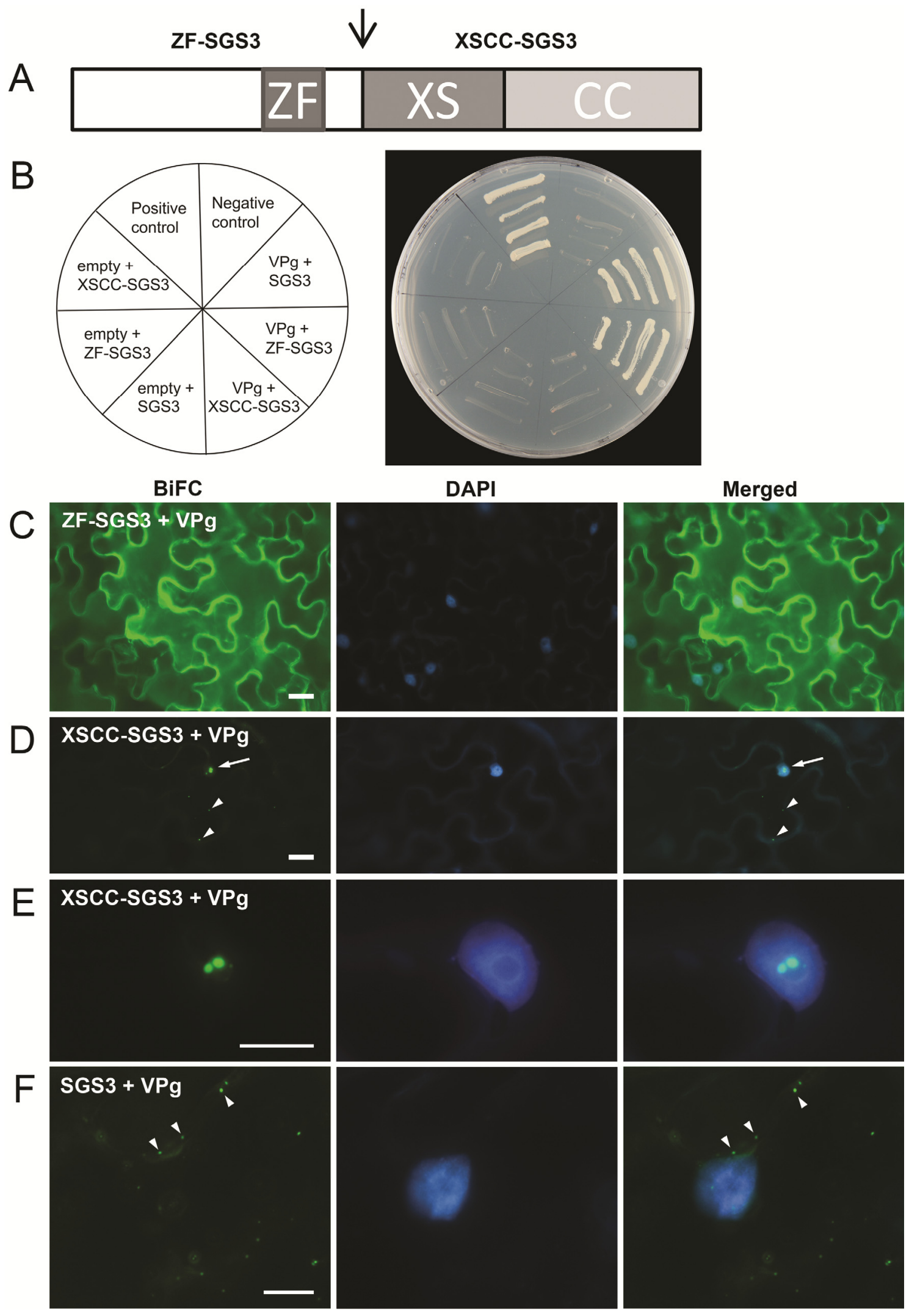

Fig. 5. Interaction of Potato virus A (PVA) viral genome-linked protein (VPg) with different domains of SGS3. A, Schematic representation of SGS3. ZF = putative zinc finger; XS = XS domain; CC = coiled coil domain. The arrow indicates where ZF-SGS3 ends and XSCC-SGS3 begins. B, Interactions of VPg with AtSGS3 and ZF-AtSGS3 and a lack of interaction with XSCC-AtSGS3 are shown via the yeast two-hybrid system (2 days growth on selective media, SD-WHAL, is shown). C, Interaction of ZF-StSGS3-VPg distributes evenly in the cytoplasm, as detected based on bimolecular fluorescence complementation (BiFC) signal in epidermal cells of leaves of Nicotiana benthamiana by epifluorescence microscope 3 days postinfiltration. D to E, Instead, interaction of XSCC-StSGS3-VPg is mainly localized to subnuclear bodies and F, interaction of full-length StSGS3-VPg localized to small bodies in the cytoplasm. DAPI (4',6-diamidino-2-phenylindole) staining indicates nuclei positions (arrows). Arrowheads point out some of the cytoplasmic granules. $\mathrm{C}$ and $\mathrm{D}$, scale bar $=20 \mu \mathrm{m}, \mathrm{E}$ and $\mathrm{F}$, scale bar $=10 \mu \mathrm{m}$. 
of gene silencing in the leaves (Ratcliff et al. 2001). The vector containing no insert (TRV-0) was used as an additional control. Inoculation was carried out as described by Rajamäki and Valkonen (2009). The plants inoculated with TRV-PDS showed a characteristic photobleaching phenotype in the upper noninoculated leaves at $14 \mathrm{dpi}$, whereas the plants inoculated with TRV-sgs3 showed a mild downward curling of the upper leaves and were stunted, as compared with the control plants inoculated with TRV-0 (Fig. 7). The symptoms were likely due to reduced SGS3 levels that accelerate transition from juvenile to adult phase (Peragine et al. 2004). Real-time RT-PCR analysis confirmed that $s g s 3$ mRNA levels were reduced in most of the TRV-sgs 3 silenced leaves as compared with leaves infected with TRV-0. However, in some cases silencing was not very efficient, which might be due to virus-induced gene silencing (VIGS) of the silencing component sgs 3 itself.

Upper leaves of the SGS3-silenced plants and the leaves at the corresponding positions of control plants were inoculated with PVA-GFP by agroinfiltration and were analyzed for accumulation of PVA CP antigen by DAS-ELISA with virus-specific monoclonal antibodies, including known amounts of PVA virions for comparison. PVA accumulated in similar amounts in the SGS3-silenced $(943 \pm 694 \mu \mathrm{g}$ of PVA CP per gram of fresh weight of leaf tissue) and control leaves (926 $\pm 234 \mu \mathrm{g}$ ) at $3 \mathrm{dpi}$, tested in three independent experiments. All plants became systemically infected with PVA. However, viral RNA levels appeared slightly reduced in the leaves expressing lower levels of SGS3 compared with control leaves, as analyzed by real-time RT-PCR (Fig. 7E). Statistical analysis of $s g s 3$ mRNA and viral RNA levels confirmed that the levels were positively correlated (Pearson correlation, $r=0.72 ; P=0.01$ ).

Subsequently, the effect of SGS3 overexpression was analyzed by agroinfiltrating the leaves of $N$. benthamiana simultaneously for expression of SGS3 and PVA. Real-time RT-PCR analysis indicated that increases in the amounts of sgs 3 mRNA and PVA RNA were correlated (Fig. 7F), consistent the data of the SGS3 silencing experiment.

\section{DISCUSSION}

Results of this study indicate that PVA VPg, a suppressor of sense-mediated RNA silencing, interacts with the SGS3 protein involved in RNA silencing. Interactions of VPg and SGS3 occurred in small cytoplasmic bodies that also contained RDR6 and likely represent the previously described SGS3/RDR6 bodies (Kumakura et al. 2009). These bodies were different from the replication vesicles that are induced by the potyviral $6 \mathrm{~K} 2$ protein and serve as the sites of potyviral multiplication. These findings are novel and potentially important because there seem to be no previous reports demonstrating interactions between the potyviral RNA silencing suppressor protein VPg and the host RNA silencing machinery. Furthermore, SGS3 has not been known to interact with silencing suppressor proteins of positive-strand RNA viruses. Results of this study also showed that SGS3 has more sites controlling homodimerization than previously known (Elmayan et al. 2009). The ZF domain of SGS3, which has been poorly understood, seems to have a role in controlling localization of SGS3 in plant cells.

PVA VPg was able to interact with the SGS3 of A. thaliana and $S$. tuberosum, as shown by our YTHS and BiFC analyses. Previously, two viral suppressors of RNA silencing, the V2 protein of TYLCV (genus Begomovirus, ssDNA genome) and the p2 protein of RSV (genus Tenuivirus, negative-sense ssRNA virus), were found to interact with SGS3 (Du et al. 2011; Glick et al. 2008). Interaction of V2 with tomato SGS3 appears necessary for suppression of RNA silencing because the mutations (C84S and $\mathrm{C} 86 \mathrm{~S}$ ) in $\mathrm{V} 2$, which compromise suppression of RNA silencing, also inhibit binding to SGS3 (Glick et al. 2008). On the other hand, another study found no interaction between V2 and SGS3 but showed that V2 competes with SGS3 in binding dsRNA substrates, which the mutant of V2 (C84S and C86S) was unable to do (Fukunaga and Doudna 2009). Therefore, it was suggested that V2 might suppress RNA silencing by competing away substrate RNAs from SGS3 rather than binding directly to SGS3 (Fukunaga and Doudna 2009). It has not been reported whether or not p2 of RSV interacts with SGS3 to suppress RNA silencing (Du et al. 2011). Our studies could not show a clear, pivotal role for the VPg-SGS3 interaction in the suppression of RNA silencing. However, because the RNA silencing suppressor proteins of unrelated viruses interact with SGS3, it seems plausible that the interaction has functional importance. Synthesis of dsRNA by RDR6 is needed for the initiation of sense-mediated RNA silencing (i.e., silencing induced by (+)ssRNA) and for the amplification of the RNA silencing response (Vaucheret 2006; Voinnet 2008). These mechanisms require SGS3 for yet-

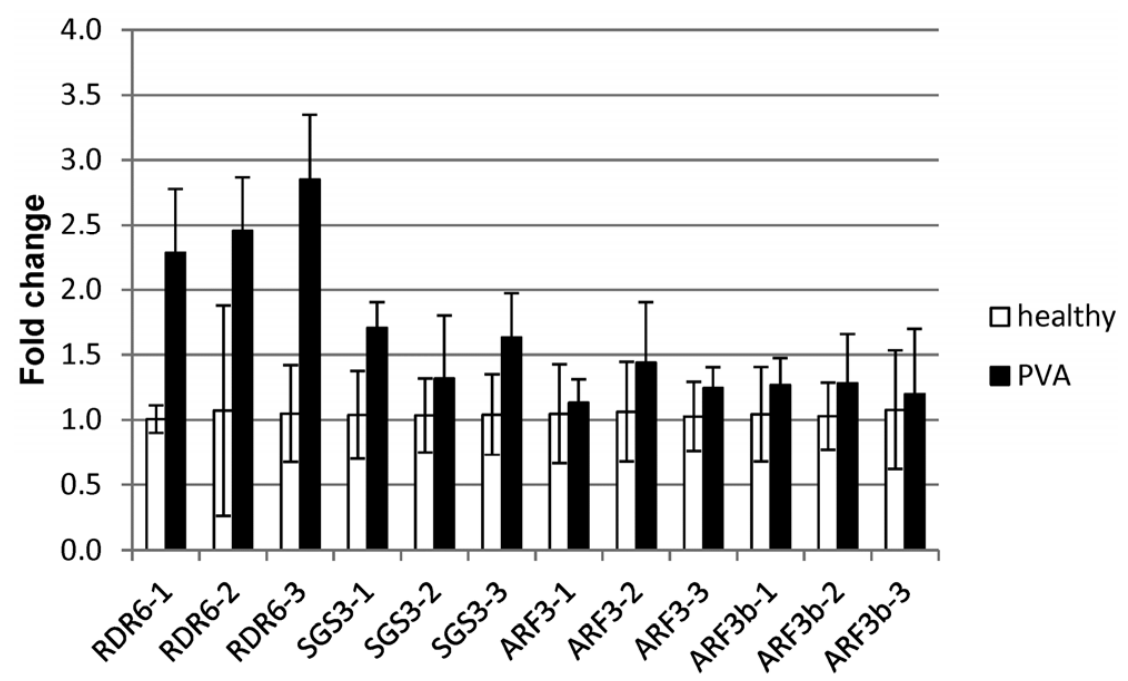

Fig. 6. Quantitative reverse transcription-polymerase chain reaction used to compare expression of RDR6, SGS3, and the putative target gene ARF3 of TAS3 trans-acting small interfering RNAs in healthy and Potato virus A (PVA)-infected tobacco (Nicotiana tabacum) plants. Phos $2 \mathrm{~A}$ was used for an internal control for data normalization. Numbers at the end of the gene names indicate the three independent experiments. Two different sets of primers were used to analyze ARF3 gene expression. Error bars show standard deviations. 

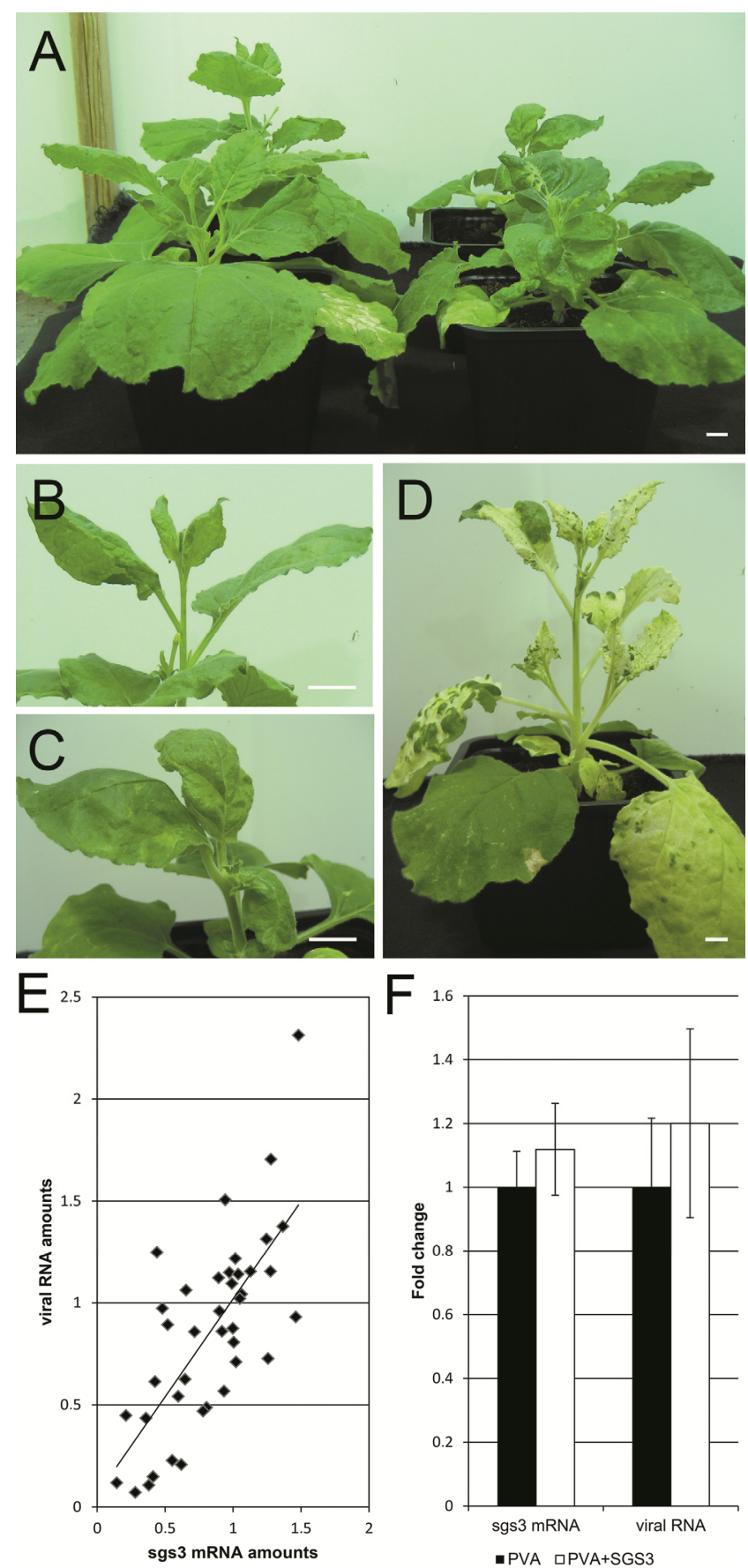

Fig. 7. Virus-induced gene silencing (VIGS) of SGS3 using Tobacco rattle virus (TRV). A, Plants of Nicotiana benthamiana systemically infected with TRV-0 vector containing no insert (right) and with TRVsgs3, which carries a 515-nucleotide-long fragment of the sgs3 gene (left) three weeks after inoculation. The plant infected with TRV-sgs 3 shows decelerated growth and mild curling of the upper leaves, unlike the plant infected with TRV-0. B, Close-up of the upper leaves of TRV-0infected plant and $\mathbf{C}$, of TRV-sgs3-infected plant. D, Silencing of the phytoene desaturase (PDS) gene in the plant infected with TRV-PDS. Scale bars $=1 \mathrm{~cm}$. E, Expression levels of $s g s 3 \mathrm{mRNA}$ and PVA RNA showed positive correlation $(r=0.72)$ in the leaves of TRV-sgs3infected and TRV-0-infected plants as analyzed by quantitative reversetranscription polymerase chain reaction (RT-PCR). The correlation was statistically significant $(P=0.01)$. Results are from three independent experiments (a total of 40 plants were analyzed). F, Mean expression levels of sgs 3 mRNA and PVA RNA were increased in leaves overexressing SGS3 when compared with control leaves as analyzed by quantitative RT-PCR. Results are from two independent experiments $(n=12$ in each experiment). $t$-Tests: $P=0.05$ for $s g s 3 \mathrm{mRNA}$ and $P=0.02$ for PVA RNA. unknown reasons. SGS3 protects the cleavage fragments of TAS2 and TAS1a transcripts from degradation after miRNAmediated cleavage during ta-siRNA biogenesis (Yoshikawa et al. 2005, 2013). However, production of Cucumber mosaic virus secondary siRNAs does not require slicing of viral RNA templates by AGO1 or AGO2 (Wang et al. 2011) suggesting that the antiviral pathway may differ from the ta-siRNA pathway (Pumplin and Voinnet 2013). PVA VPg, TYLCV V2, and RSV p2 suppress only sense-mediated RNA silencing and cannot suppress silencing induced by dsRNA, e.g., the so-called hairpin RNA (Du et al. 2011; Germundsson et al. 2007; Glick et al. 2008; Rajamäki and Valkonen 2009; Zrachya et al. 2007). Therefore, it seems likely that these viral proteins target a step of RNA silencing that involves synthesis of dsRNA, although this hypothesis is yet to be tested experimentally.

During the review process of this paper, the triple gene block protein (TGBp1) of Plantago asiatica mosaic virus (PlAMV; genus Potexvirus), which is a silencing suppressor protein, was reported to interact with both SGS3 and RDR6 (Okano et al. 2014). TGBp1 appears to mediate aggregation of SGS3 and RDR6 via homo-oligomerization of the viral protein, which leads to inhibition of dsRNA synthesis. However, VPgSGS3 interaction bodies remained dispersed in the cytoplasm, which suggests a different mechanism.

The small cytoplasmic SGS3/RDR6 bodies reported previously appear to be sites for dsRNA synthesis and also house the interactions we observed between VPg and SGS3 (Jouannet et al. 2012; Kumakura et al. 2009). The VPg-SGS3 interaction bodies detected in virus-infected cells in our study were different from the $6 \mathrm{~K} 2-\mathrm{mRFP}$-induced vesicles that are proposed to be the sites of virus replication (Beauchemin et al. 2007; Cotton et al. 2009; Wei et al. 2010). Our results differ from a previous study that reported partial overlap of the siRNA bodies and the $6 \mathrm{~K} 2$-derived vesicles, which were induced by overexpression of $6 \mathrm{~K} 2$ rather than by viral infection (Jouannet et al. 2012). VPg shows a strong tendency for nuclear localization and, hence, VPg-host protein interactions also tend to be observed in the nucleus. However, 6K2 anchors the 6K2-NIa (precursor protein of VPg) complex to cytoplasmic membranes, which impedes nuclear targeting of the precursor protein (RestrepoHartwig and Carrington 1992, 1994; Schaad et al. 1997). Therefore, protein-protein interactions with the VPg precursor protein are detected in the cytoplasm (Beauchemin and Laliberté 2007; Beauchemin et al. 2007; Huang et al. 2010; Thivierge et al. 2008). The observed subcellular localization of the VPg-SGS3 interaction in plant cells suggests that SGS3 retains VPg in the cytoplasm, perhaps because the SGS3/RDR6 bodies associate with membranes (Jouannet et al. 2012). Alternatively, it is possible that the VPg-SGS3 interaction masks the NLS of VPg. This latter idea is supported by the slightly weaker interactions between SGS3 and the NLS mutants of $\mathrm{VPg}$, indicating that the NLS regions contributed to interactions with SGS3.

SGS3 functions as a homodimer (Elmayan et al. 2009) and contains three major domains. The XS and $\mathrm{CC}$ are required for granule-like localization of SGS3 (Kumakura et al. 2009), which was also supported by our study. Furthermore, the XS domain is needed for RNA binding (Fukunaga and Doudna 2009; Zhang and Trudeau 2008) and the CC domain is involved in SGS3 homodimerization (Elmayan et al. 2009). The functions of the ZF domain have not been elucidated, but our data indicate that the ZF domain may also contribute to homodimerization by interacting with itself and the XS and $\mathrm{CC}$ domains. These new data suggest that multiple protein domains control SGS3 self-interactions. Furthermore, results indicated that the ZF of SGS3 largely mediated the SGS3-VPg interaction. ZF domains can function in protein 
recognition, although their role in DNA-binding is better understood (Gamsjaeger et al. 2007). It has not yet been reported which domain of SGS3 controls interactions with TYLCV V2 (Glick et al. 2008), whereas it is known that multiple regions of SGS3 can interact with RSV p2 (Du et al. 2011). Our studies also show that ZF affects the localization of SGS3. This was especially obvious with the VPg-SGS3 interaction. The truncated SGS3 lacking a ZF domain could interact with VPg, but the interaction was observed almost exclusively in the nucleus and various subnuclear structures, whereas interactions of VPg with the full-length SGS3 were found to localize to cytoplasmic bodies. These data point out that the ZF domain of SGS3 is pivotal for the proper cytoplasmic localization of VPg-SGS3 interactions.

Mutations in the NLS of VPg slightly reduced the strength of the VPg-SGS3 interaction but did not abolish it. The same mutations abolish PVA infectivity and the silencing suppressor ability of VPg (Rajamäki and Valkonen 2009). Whether the SGS3-VPg interaction is required for these functions remains to be studied, as the same viral regions also control nuclear and nucleolar localization of VPg and NIa (Rajamäki and Valkonen 2009) and NTP-binding and uridylylation of VPg in one of the regions (Rantalainen et al. 2010).

The SGS3-silenced plants of $N$. benthamiana were infected systemically with PVA and accumulation of viral RNA appeared correlated with the expression level of $s g s 3$. Viral RNA levels were slightly reduced in the SGS3-silenced leaves of $N$. benthamiana when compared with controls, although no differences in viral CP levels could be detected by ELISA. In addition, overexpression of SGS3 resulted in enhanced viral RNA accumulation. These results suggest that SGS3 has a role in PVA infection and may also propose that the interaction of VPg with SGS3 further protects viral RNA from degradation. A previous study has suggested that VPg may increase the stability of viral RNA (Eskelin et al. 2011). The hypothesis was based on the findings that VPg coexpression with PVA increases viral translation and accumulation of viral RNA, even in the case of a replicative-deficient PVA mutant (Eskelin et al. 2011). SGS3 allows stabilization of TAS transcripts, which protects them from degradation (Yoshikawa et al. 2005, 2013), and, given the observed interactions between VPg and SGS3, it is possible that VPg recruits SGS3 to protect viral RNA. Alternatively, the effect may be due to interference in the amplification step of RNA silencing via a yet-unidentified mechanism. The two processes may be also interlinked. Recent data indicate that sense-mediated RNA silencing is intimately linked to other RNA quality control systems and that SGS3/RDR6 bodies may exchange substrates with $\mathrm{P}$ bodies under some circumstances (Moreno et al. 2013; Voinnet 2008). It is, thus, possible that VPg functions in both RNA silencing and in other RNA quality control systems. In contrast to our data, Cucumber mosaic virus (genus Cucumovirus) shows enhanced accumulation of viral RNA in sgs3 mutant Arabidopsis plants as compared with wild-type plants (Mourrain et al. 2000). In addition, a mutant of Cucumber mosaic virus, deficient in silencing suppression, which is predominantly targeted for silencing via the RDR6-dependent pathway (Wang et al. 2010), fails to infect wild-type Arabidopsis but is highly virulent in $s g s 3$ mutant plants (Wang et al. 2011). The data thus suggest that the impact of SGS3 interaction varies among plant viruses.

Our previous study showed that PVA VPg plays a role in interference with RNA silencing (Rajamäki and Valkonen 2009). The data presented here provides further links between VPg and RNA silencing, showing that VPg interacts with SGS3, a component of the antiviral RNA-silencing pathway. The data also suggest that viruses may interact with SGS3 for different purposes.

\section{MATERIALS AND METHODS}

Plant material.

$N$. tabacum cv. Samsun nn and N. benthamiana plants were grown from seeds. Potato plants ( $S$. tuberosum line $2 \times v 2(2-7)$ [Valkonen et al. 1994]) were obtained from our in vitro collection. Plants were grown in growth chambers under constant conditions (16-h photoperiod at 18 and $22^{\circ} \mathrm{C}, 40 \%$ relative humidity; $200 \mu \mathrm{E} \mathrm{m}^{-2} \mathrm{~s}^{-1}$ light intensity) and were watered with fertilizer (N/P/K = 16:9:22; Yara, Espoo, Finland) mixed in water $(0.3 \mathrm{~g} /$ liter $)$ given at each watering.

\section{YTHS.}

AtSGS3 (At5g23570) and the deletion mutants of AtSGS3 (ZF-SGS3, aa 1 to 289 and XSCC-SGS3, aa 290 to 625) were amplified with PCR from a plasmid obtained from Nottingham Arabidopsis Stock Center, using Phusion high-fidelity DNA polymerase (Finnzymes, Espoo, Finland) and primers designed based on the published sequence (Supplementary Table 1). AtRDR6 was amplified from a plasmid, pBIC-AtRDR6smGFP (provided by Y. Watanabe [Kumakura et al. 2009]). The amplification products were cloned in-frame with the GAL4 DNA BD and AD in the vectors pGBKT7 and pGADT7, respectively (Matchmaker Gal4 Yeast Two-Hybrid System 3; Clontech, Mountain View, CA, U.S.A.) and were verified by sequencing. Cloning of PVA VPg and NLS mutants of VPg has been described previously (Rajamäki and Valkonen 2009). $\operatorname{VPg}(\mathrm{a})$ (K4A, R5A, R7A, and K9A), and $\mathrm{VPg}(\mathrm{b})$ (K41A, K42A, K44A, K46A, and K48A) mutants contain four and five amino acid substitutions in NLS I and NLS II in VPg, respectively, and $\mathrm{VPg}(\mathrm{ab})$ contains the combination of mutations of both $\operatorname{VPg}(a)$ and $\operatorname{VPg}(b)$. PVA NIa(E/H), which contains the mutation $(\mathrm{E} 189 \mathrm{H})$ that prevents internal proteolytic processing between VPg and NIa-Pro, was cloned similarly, using pRT-GFP-NIa(E/H) as a template (Rajamäki and Valkonen 2009). Site-directed mutagenesis of the amino acids (C228A and $\mathrm{C} 231 \mathrm{~A}$ ) in the $\mathrm{ZF}$ of AtSGS3 was carried out using the QuickChange II XL site-directed mutagenesis system (Stratagene, La Jolla, CA, U.S.A.) and appropriate nucleotide primers.

Saccharomyces cerevisiae (strain AH109) was co-transformed with the $\mathrm{BD}$ and $\mathrm{AD}$ plasmids and protein-protein interactions were detected by yeast growth on medium lacking adenine, histidine, leucine, and tryptophan at $30^{\circ} \mathrm{C}$ for up to 10 days, as described (Clontech). Additionally, yeast growth was studied on the selective medium with $5 \mathrm{mM} 3$-AT added for additional stringency.

To verify fusion protein expression in yeast, total protein was extracted, and Western blot analysis using monoclonal AD and BD antibodies (Clontech, Mountain View, CA, U.S.A.) was carried out as described previously (Ala-Poikela et al. 2011; Volland et al. 1994).

\section{BiFC and fluorescently labeled proteins.}

Total RNA was extracted using Trizol made in-house (Caldo et al. 2004) from potato leaves, line $2 \times v 2(2-7)$, and cDNA was synthesized using PowerScript reverse transcriptase (Clontech) and a poly $\mathrm{T}+\mathrm{N}$-primer. The potato $S G S 3$ genes ( $S t S G S 3$ a, $S t S G S 3$ b) were amplified by PCR from cDNA, using designed primers based on potato genome sequence information (Potato Genome Sequencing Consortium, Solanum tuberosum Group Phureja DM v3.4 CDS sequences) and containing restriction sites needed for cloning. AtSGS3, AtSGS3-ZFmut, and AtRDR6 genes and VPg encoding region were amplified from corresponding YTHS plasmids. The truncated versions of StSGS3 (ZF-StSGS3 aa 1 to 292 and XSCC-StSGS3 aa 293 to 631) were amplified from the previously cloned StSGS3 BiFC plasmid. The PCR products were cloned in frame with the N-proxi- 
mal (YN) and the C-proximal (YC) halves of the YFP gene in the binary expression cassettes described by Zamyatnin and associates (2006) and were verified by sequencing. Binary vectors were introduced into Agrobacterium tumefaciens (pGV2260) cells by electroporation using a Bio-Rad Gene Pulser. Agrobacterium cultures were diluted with the induction medium (10 $\mathrm{mM} \mathrm{MgCl}_{2}$ and $20 \mu \mathrm{M}$ acetosyringone), were combined in equal volumes to final optical densities at $600 \mathrm{~nm}$ $\left(\mathrm{OD}_{600}\right)=0.25$ to 0.3 , and were agroinfiltrated into leaves of $N$. benthamiana as previously described (Llave et al. 2000).

The vector for expressing AtRDR6-GFP (pBIC-AtRDR6smGFP) was provided by Y. Watanabe, University of Tokyo, Japan. pLH-AtRDR6-mRFP was constructed by releasing the 6K2-encoding fragment from pLH-6K2-mRFP (Ala-Poikela et al. 2011) by digestion with XhoI, followed by the amplification and ligation of AtRDR6. The construct was verified by sequencing.

\section{Fluorescence microscopy.}

Epifluorescence microscopy was performed using a Leitz Laborlux $\mathrm{S}$ microscope with an epifluorescence extension Leitz Ploemopak (Leica Microsystems GmbH, Wetzlar, Germany) and appropriate filters (excitation 470/40 nm, emission $525 / 50 \mathrm{~nm}$ ) for visualization of YFP. Images were captured using the charge-coupled device camera DP-50 (Olympus, Tokyo), which was controlled by Viewfinder Lite, software version 1.0 (Olympus). Alternatively, a Zeiss epifluorescence microscope (Axioimager M2, Carl Zeiss Microscopy GmbH, Jena, Germany) and a YFP-compatible filter cube (GFP) were used.

Confocal microscopy was performed using a Leica TCS SP2 AOBS device and $\times 63$ water immersion objective at the Institute of Biotechnology, University of Helsinki. Emission from GFP and RFP were scanned sequentially as follows: GFP was excited with argon laser at $488 \mathrm{~nm}$ and was captured at 500 to $550 \mathrm{~nm}$, and RFP was excited with a 561-nm DPSS laser and was captured at 600 to $640 \mathrm{~nm}$. YFP resulting from BiFC was excited with a 514-nm argon laser and was captured at 520 to $580 \mathrm{~nm}$.

\section{Construction of recombinant PVA and viral detection.}

To construct PVA-vpgyc, VPg-YC was amplified from the corresponding BiFC construct using appropriate primers including sites for $B f r \mathrm{I}$ and $M l u \mathrm{I}$ and was cloned into the $\mathrm{NIb}$ and CP junction of the infectious clone of PVA-B11 (Kelloniemi et al. 2008). For agroinfiltration, the clone was transferred to the modified binary vector pCAMBIA0390 (Cambia, Brisbane, Australia) using KpnI and SalI sites as previously described (Ala-Poikela et al. 2011). The constructs were verified by restriction enzyme analysis and sequencing. Construction of a binary PVA-6krfp clone has been described (Ala-Poikela et al. 2011).

PVA was detected with DAS-ELISA, using a monoclonal antibody specific to PVA (MAb 58/0) and the alkaline phosphatase-conjugated MAb 58/0 (SASA, Edinburgh, U.K.) as previously described (Rajamäki et al. 1998). Leaf samples were weighed, ground in ELISA sample buffer at $1 \mathrm{~g}$ to $10 \mathrm{ml}$, and were subsequently diluted 100-fold. Two 100- $\mu$ l aliquots were transferred to an ELISA microtiter plate coated with PVA antibodies. Known amounts of purified PVA virion were included for comparison to estimate virus concentration.

\section{Quantitative RT-PCR.}

Plants of N. tabacum (BBCH growth stage code 1004) were sap-inoculated with PVA as described by Haikonen and associates (2013). Total RNA was extracted from the leaves systemically infected with PVA, RNA $(2 \mu \mathrm{g})$ was DNase treated, and first-strand cDNA synthesis carried out using Moloney murine leukemia virus reverse transcriptase (Promega) and random primers as described (Haikonen et al. 2013). Quantitative PCR was carried out using Light Cycler 480 SYBR Green I PCR master mix (Roche, Mannheim, Germany) and the Light Cycler 480 real time PCR detection system (Roche). Primers were designed for SGS3, RDR6, and ARF3. Phos2A was used for an internal control for data normalization. PCR amplification efficiency and relative gene expression were calculated by setting the threshold with the second derivative maximum and calculating fold changes with the E method as previously described (Rajamäki and Valkonen 2009).

\section{VIGS and SGS3 overexpression.}

A 515-base pair fragment from the middle portion of tobacco SGS3 was amplified by PCR from tobacco cDNA, cloned into the SmaI site of TRV RNA2 vector pTV00 (Ratcliff et al. 2001), and the construct (pTV:sgs3) was transformed into A. tumefaciens GV3101. To induce VIGS, A. tumefaciens strains carrying TRV RNA1 (pBINTRA6) and RNA2 (pTV:00, pTV:sgs3, or pTV:PDS) were combined in a ratio of $1: 1(\mathrm{vol} / \mathrm{vol})$ and were agroinfiltrated to leaves of $N$. benthamiana, as described (Rajamäki and Valkonen 2009).

The upper leaves of TRV-sgs3-infected plants and the corresponding leaves from plants infected with TRV-0 were inoculated three weeks after the induction of VIGS with GFP-tagged PVA by agroinfiltration, using the final $\mathrm{OD}_{600}$ of 0.5 to achieve uniform infection of a large number of cells. The inoculated leaves were collected $3 \mathrm{dpi}$ and were analyzed for PVA CP amounts with DAS-ELISA, as described above. Alternatively, wild-type plants of $N$. benthamiana were agroinfiltrated for overexpression of SGS3 simultaneously with PVA and SGS3YC or with PVA only as a control, using the final $\mathrm{OD}_{600}$ of 0.5 . The inoculated leaves were collected for analysis $3 \mathrm{dpi}$. Amounts of viral RNA and expression of sgs 3 mRNA were analyzed by quantitative RT-PCR as described above from all plants. Correlation between sgs3 mRNA and viral RNA amounts were analyzed statistically, using Pearson correlation. sgs3 mRNA and viral RNA levels were statistically analyzed from SGS3 overexpression experiments using a $t$-test.

\section{ACKNOWLEDGMENTS}

We thank Y. Watanabe (University of Tokyo, Japan) for providing pBIC-AtRDR6-smGFP and T. Haikonen for analysis of tobacco microarray data. Financial support from the Academy of Finland (grant 1253126 to J. P. T. Valkonen.) is gratefully acknowledged.

\section{LITERATURE CITED}

Adams, M. J., Zerbini, F. M., French, R., Rabenstein, F., Stenger, D. C., and Valkonen, J. P. T. 2012. Family Potyviridae. Pages 1069-1089 in: Virus Taxonomy, Ninth Report of the International Committee on Taxonomy of Viruses, A. M. Q. King, M. J. Adams, E. B. Carstens, and E. J. Lefkowitz, eds. Elsevier Academic Press, London, U.K.

Ala-Poikela, M., Goytia, E., Haikonen, T., Rajamäki, M. L., and Valkonen, J. P. T. 2011. Helper component proteinase of the genus Potyvirus is an interaction partner of translation initiation factors $\mathrm{IF}$ (iso)4E and eIF4E and contains a 4E binding motif. J. Virol. 85:6784-6794.

Anandalakshmi, R., Pruss, G. J., Ge, X., Marathe, R., Mallory, A. C., Smith, T. H., and Vance, V. B. 1998. A viral suppressor of gene silencing in plants. Proc. Natl. Acad. Sci. U.S.A. 95:13079-13084.

Axtell, M. J., Jan, C., Rajagopalan, R., and Bartel, D. P. 2006. A two-hit trigger for siRNA biogenesis in plants. Cell 127:565-577.

Azevedo, J., Garcia, D., Pontier, D., Ohnesorge, S., Yu, A., Garcia, S., Braun, L., Bergdoll, M., Hakimi, M. A., Lagrange, T., and Voinnet, O. 2010. Argonaute quenching and global changes in Dicer homeostasis caused by a pathogen-encoded GW repeat protein. Genes Dev. 24:904915.

Beauchemin, C., and Laliberté, J.-F. 2007. The poly(A) binding protein is internalized in virus-induced vesicles or redistributed to the nucleolus 
during Turnip mosaic virus infection. J. Virol. 81:10905-10913.

Beauchemin, C., Boutet, N., and Laliberté, J.-F. 2007. Visualization of the interaction between the precursors of $\mathrm{VPg}$, the viral protein linked to the genome of Turnip mosaic virus, and the translation eukaryotic initiation factor iso 4E in planta. J. Virol. 81:775-782.

Béclin, C., Boutet, S., Waterhouse, P., and Vaucheret, H. 2002. A branched pathway for transgene-induced RNA silencing in plants. Curr. Biol. 12:684-688.

Bortolamiol, D., Pazhouhandeh, M., Marrocco, K., Genschik, P., and Ziegler-Graff, V. 2007. The polerovirus F box protein PO targets ARGONAUTE1 to suppress RNA silencing. Curr. Biol. 17:1615-1621.

Brigneti, G., Voinnet, O., Li, W.-X., Ji, L.-H., Ding, S.-W., and Baulcombe, D. C. 1998. Viral pathogenicity determinants are suppressors of transgene silencing in Nicotiana benthamiana. EMBO (Eur. Mol. Biol. Organ.) J. 17:6739-6746.

Caldo, R. A, Nettleton, D., and Wise, R. P. 2004. Interaction-dependent gene expression in $M l a$-specified response to barley powdery mildew. Plant Cell 16:2514-2528.

Chiu, M. H., Chen, I. H., Baulcombe, D. C., and Tsai, C. H. 2010. The silencing suppressor P25 of Potato virus $X$ interacts with Argonaute1 and mediates its degradation through the proteasome pathway. Mol. Plant Pathol. 11:641-649.

Cotton, S., Grangeon, R., Thivierge, K., Mathieu, I., Ide, C., Wei, T. Wang, A., and Laliberté, J.-F. 2009. Turnip mosaic virus RNA replication complex vesicles are mobile, align with microfilaments, and are each derived from a single viral genome. J. Virol. 83:1046010471.

Du, Z., Xiao, D., Wu, J., Jia, D., Yuan, Z., Liu, Y., Hu, L., Han, Z., Wei, T., Lin, Q., Wu, Z., and Xie, L. 2011. p2 of Rice stripe virus (RSV) interacts with OsSGS3 and is a silencing suppressor. Mol. Plant Pathol. 12:808-814

Elmayan, T., Adenot, X., Gissot, L., Lauressergues, D., Gy, I., and Vaucheret, H. 2009. A neomorphic sgs3 allele stabilizing miRNA cleavage products reveals that SGS3 acts as a homodimer. FEBS (Fed. Eur. Biochem. Soc.) J. 276:835-844.

Eskelin, K., Hafren, A., Rantalainen, K., and Mäkinen, K. 2011. Potyviral VPg enhances viral RNA translation and inhibits reporter mRNA translation in planta. J. Virol. 85:9210-9221.

Fukunaga, R., and Doudna, J. A. 2009. dsRNA with 5' overhangs contributes to endogenous and antiviral RNA silencing pathways in plants. EMBO (Eur. Mol. Biol. Organ.) J. 28:545-555.

Gamsjaeger, R., Liew, C. K., Loughlin, F. E., Crossley, M., and Mackay, J. P. 2007. Sticky fingers: Zinc-fingers as protein recognition motifs. Trends Biochem. Sci. 32:63-70.

Germundsson, A., Savenkov, E. I., Ala-Poikela, M., and Valkonen, J. P. T. 2007. VPg of Potato virus A alone does not suppress RNA silencing but affects virulence of a heterologous virus. Virus Genes 34:387-399.

Giner, A., Lakatos, L., Garcia-Chapa, M., Lopez-Moya, J. J., and Burgyan, J. 2010. Viral protein inhibits RISC activity by Argonaute binding through conserved WG/GW motifs. PLoS Pathog. 6:e1000996. Published online.

Glick, E., Zrachya, A., Levy, Y., Mett, A., Gidoni, D., Belausov, E., Citovsky, V., and Gafni, Y. 2008. Interaction with host SGS3 is required for suppression of RNA silencing by tomato yellow leaf curl virus V2 protein. Proc. Natl. Acad. Sci. U.S.A. 105:157-161

Gonzalez, I., Martinez, L., Rakitina, D. V., Lewsey, M. G., Atencio, F. A., Llave, C., Kalinina, N. O., Carr, J. P., Palukaitis, P., and Canto, T. 2010. Cucumber mosaic virus $2 \mathrm{~b}$ protein subcellular targets and interactions: Their significance to RNA silencing suppressor activity. Mol. PlantMicrobe Interact. 23:294-303.

Guo, H., Song, X., Xie, C., Huo, Y., Zhang, F., Chen, X., Geng, Y., and Fang, R. 2013. Rice yellow stunt rhabdovirus protein 6 suppresses systemic RNA silencing by blocking RDR6-mediated secondary siRNA synthesis. Mol. Plant-Microbe Interact. 26:927-936.

Haikonen, T., Rajamäki, M.-L., Tian, Y.-P., and Valkonen, J. P. T. 2013. Mutation of a short variable region in HCpro protein of Potato virus A affects interactions with a microtubule-associated protein and induces necrotic responses in tobacco. Mol. Plant-Microbe Interact. 26:721-733.

Himber, C., Dunoyer, P., Moissiard, G., Ritzenthaler, C., and Voinnet, O. 2003. Transitivity-dependent and -independent cell-to-cell movement of RNA silencing. EMBO (Eur. Mol. Biol. Organ.) J. 22:4523-4533.

Huang, T.-S., Wei, T., Laliberté, J.-F., and Wang, A. 2010. A host RNA helicase-like protein, AtRH8, interacts with the potyviral genomelinked protein, VPg, associates with the virus accumulation complex, and is essential for infection. Plant Physiol. 152:255-266.

Jamous, R. M., Boonrod, K., Fuellgrabe, M. W., Ali-Shtayeh, M. S., Krczal, G., and Wassenegger, M. 2011. The helper component-proteinase of the Zucchini yellow mosaic virus inhibits the Hua Enhancer 1 methyltransferase activity in vitro. J. Gen. Virol. 92:2222-2226.

Jouannet, V., Moreno, A. B., Elmayan, T., Vaucheret, H., Crespi, M. D., and Maizel, A. 2012. Cytoplasmic Arabidopsis AGO7 accumulates in membrane-associated siRNA bodies and is required for ta-siRNA biogenesis. EMBO (Eur. Mol. Biol. Organ.) J. 31:1704-1713.

Joung, J. K., Ramm, E. I., and Pabo, C. O. 2000. A bacterial two-hybrid selection system for studying protein-DNA and protein-protein interactions. Proc. Natl. Acad. Sci. U.S.A. 97:7382-7387.

Kasschau, K. D., and Carrington, J. C. 1998. A counterdefensive strategy of plant viruses: Suppression of posttranscriptional gene silencing. Cell 95:461-470.

Kelloniemi, J., Mäkinen, K., and Valkonen, J. P. T. 2008. Three heterologous proteins simultaneously expressed from a chimeric potyvirus: Infectivity, stability, and the correlation of genome and virion length. Virus Res. 135:282-291.

Kumakura, N., Takeda, A., Fujioka, Y., Motose, H., Takano, R., and Watanabe, Y. 2009. SGS3 and RDR6 interact and colocalize in cytoplasmic SGS3/RDR6-bodies. FEBS (Fed. Eur. Biochem. Soc.) Lett. 583:1261-1266.

Llave, C., Kasschau, K. D., and Carrington, J. C. 2000. Virus-encoded suppressor of posttranscriptional gene silencing targets a maintenance step in the silencing pathway. Proc. Natl. Acad. Sci. U.S.A. 97:1340113406.

Moreno, A. B., Martínez de Alba, A. E. M., Bardou, F., Crespi, M. D., Vaucheret, H., Maizel., A., and Mallory, A. C. 2013. Cytoplasmic and nuclear quality control and turnover of single-stranded RNA modulate post-transcriptional gene silencing in plants. Nucleic Acids Res. 41:4699-4708.

Mourrain, P. Béclin, C., Elmayan, T., Feuerback, F., Godon, C., Morel, J. B., Jouette, D., Lacombe, A. M., Nikic, S., Picault, N., Remoue, K., Sanial, M., Vo, T. A., and Vaucheret, H. 2000. Arabidopsis SGS2 and $S G S 3$ genes are required for posttranscriptional gene silencing and natural virus resistance. Cell 101:533-542.

Okano, Y., Senshu, H., Hashimoto, M., Neriya, Y., Netsu, O., Minato, N., Yoshida, T., Maejima, K., Oshima, K., Komatsu, K., Yamaji, Y., and Namba, S. 2014. In planta recognition of a double-stranded RNA synthesis protein complex by a potexviral RNA silencing suppressor. Plant Cell (doi:10.1105/tpc.113.120535, advance on line publication).

Peragine, A., Yoshikawa, M, Wu, G., Albrecht, H. L., and Poethig, R. S. 2004. SGS3 and SGS2/SDE1/RDR6 are required for juvenile development and the production of trans-acting siRNAs in Arabidopsis. Genes Dev. 18:2368-2379.

Pontes, O., Vitins, A., Ream, T. S., Hong, E., Pikaard, C. S., and CostaNunes, P. 2013. Intersection of small RNA pathways in Arabidopsis thaliana sub-nuclear domains. PLoS One 8:e65652. Published online.

Pumplin, N., and Voinnet, O. 2013. RNA silencing suppression by plant pathogens: Defence, counter-defence and counter-counter-defence. Nat. Rev. Microbiol. 11:745-760.

Rajamäki, M. L., and Valkonen, J. P. T. 2009. Control of nuclear and nucleolar localization of nuclear inclusion protein A of picorna-like Potato virus A in Nicotiana species. Plant Cell 21:2485-2502.

Rajamäki, M., Merits, A., Rabenstein, F., Andrejeva, J., Paulin, L. Kekarainen, T., Kreuze, J. F., Forster, R. L. S., and Valkonen, J. P. T. 1998. Biological, serological, and molecular differences among isolates of potato a potyvirus. Phytopathology 88:311-321.

Rajamäki, M. L., Mäki-Valkama, T., Mäkinen, K., and Valkonen, J. P. T. 2004. Infection with potyviruses. Pages 68-91 in: Plant-Pathogen Interactions, N. J. Talbot, ed. Blackwell Publishing, Sheffield, U.K..

Rantalainen, K. I., Eskelin, K., Tompa, P., and Mäkinen, K. 2010. Structure flexibility allows the functional diversity of potyviral genome-linke protein VPg. J. Virol. 85:2449-2457.

Ratcliff, F., Martin-Hernandez, A. M., and Baulcombe, D. C. 2001. Tobacco rattle virus as a vector for analysis of gene function by silencing. Plant J. 25:237-245.

Restrepo-Hartwig, M. A., and Carrington, J. C. 1992. Regulation of nuclear transport of a plant potyvirus protein by autoproteolysis. J. Virol. 66:5662-5666.

Restrepo-Hartwig, M. A., and Carrington, J. C. 1994. The tobacco etch potyvirus 6-kilodalton protein is membrane associated and involved in viral replication. J. Virol. 68:2388-2397.

Schaad, M. C., Jensen, P. E., and Carrington, J. C. 1997. Formation of plant RNA virus replication complexes on membranes: Role of an endoplasmic reticulum-targeted viral protein. EMBO (Eur. Mol. Biol. Organ.) J. 16:4049-4059.

Szittya, G., and Burgyán, J. 2013. RNA interference-mediated intrinsic antiviral immunity in plants. Curr. Top. Microbiol. 371:153-181.

Thivierge, K., Cotton, S., Dufresne, P. J., Mathieu, I., Beauchemin, C., Ide, C., Fortin, M. G., and Laliberté, J.-F. 2008. Eukaryotic elongation factor 1A interacts with Turnip mosaic virus RNA-dependent RNA polymerase and VPg-Pro in virus-induced vesicles. Virology 377:216225.

Valkonen, J., Slack, S., Plaisted, R., and Watanabe, K. 1994. Extreme 
resistance is epistatic to hypersensitive resistance to potato virus $\mathrm{Y}$ in a Solanum tuberosum ssp. andigena -derived potato genotype. Plant Dis. 1994, 78:1177-1180.

Vaucheret, H. 2006. Post-transcriptional small RNA pathways in plants: Mechanisms and regulations. Genes Dev. 20:759-771.

Voinnet, O. 2008. Use, tolerance and avoidance of amplified RNA silencing by plants. Trends Plant Sci 13:317-328.

Volland, C., Urban-Grimal, D., Géraud, G., and Haguenauer-Tapis, R. 1994. Endocytosis and degradation of the yeast uracil permease under adverse condition. J. Biol. Chem. 269:9833-9841.

Wang, X. B., Wu, Q., Ito, T., Cillo, F., Li, W. X., Chen, X., Yu, J. L., and Ding, S. W. 2010. RNAi-mediated viral immunity requires amplification of virus-derived siRNAs in Arabidopsis thaliana. Proc. Natl. Acad. Sci. U.S.A. 107:484-489.

Wang, X. B., Jovel, J., Udomporn, P., Wang, Y., Wu, Q., Li, W. X., Gasciolli, V., Vaucheret, H., and Ding, S. W. 2011. The 21-nucleotide, but not 22nucleotide, viral secondary small interfering RNAs direct potent antiviral defense by two cooperative agronautes in Arabidopsis thaliana. Plant Cell 23:1625-1638.

Wei, T., Huang, T.-S., McNeil, J., Laliberté, J.-F., Hong, J., Nelson, R. S., and Wang, A. 2010. Sequential recruitment of the endoplasmic reticulum and chloroplasts for plant potyvirus replication. J. Virol. 84:799809.

Yoshikawa, M., Peragine, A., Park, M. Y., and Poethig, R. S. 2005. A path- way for the biogenesis of trans-acting siRNAs in Arabidopsis. Gene. Dev. 19:2164-2175.

Yoshikawa, M., Iki, T., Tsutsui, Y., Miyashita, K., Poethig, R. S., Haby, Y., and Ishikawa, M. 2013. 3' fragment of miR173-programmed RISCcleaved RNA is protected from degradation in a complex with RISC and SGS3. Proc. Natl. Acad. Sci. U.S.A. 110:4117-4122.

Zamyatnin, A. A., Jr., Solovyev, A. G., Bozhkov, P. V., Valkonen, J. P. T., Morozov, S. Y., and Savenkov, E. I. 2006. Assessment of the integral membrane protein topology in living cells. Plant J. 46:145-154.

Zhang, D., and Trudeau, V. L. 2008. The XS domain of a plant specific SGS3 protein adapts a unique RNA recognition motif (RRM) fold. Cell Cycle 7:2268-2270.

Zhang, X., Yuan, Y. R., Pei, Y., Lin, S. S., Tuschl, T., Patel, D. J., and Chua, N. H. 2006. Cucumber mosaic virus-encoded $2 \mathrm{~b}$ suppressor inhibits Arabidopsis Argonaute1 cleavage activity to counter plant defense. Gene Dev. 20:3255-3268.

Zrachya, A., Glick, E., Levy, Y., Arazi, T., Citovsky, V., and Gafni, Y. 2007. Suppressor of RNA silencing encoded by Tomato yellow leaf curl virusIsrael. Virology 358:159-165.

\section{AUTHOR-RECOMMENDED INTERNET RESOURCE}

Nottingham Arabidopsis Stock Centre website: www.arabidopsis.info 